\title{
In-depth characterization of the cisplatin mutational signature in human cell lines and in esophageal and liver tumors
}

\author{
Arnoud Boot, ${ }^{1,2}$ Mi Ni Huang, ${ }^{1,2}$ Alvin W.T. Ng, ${ }^{2,3}$ Szu-Chi Ho, ${ }^{1}$ jing Quan Lim, ${ }^{4,5}$ \\ Yoshiiku Kawakami, ${ }^{6}$ Kazuaki Chayama, ${ }^{6}$ Bin Tean Teh, ${ }^{7}$ Hidewaki Nakagawa, ${ }^{8}$ \\ and Steven G. Rozen ${ }^{1,2}$
}

${ }^{1}$ Cancer and Stem Cell Biology, Duke-NUS Medical School, 169857 Singapore; ${ }^{2}$ Centre for Computational Biology, Duke-NUS Medical School, 169857 Singapore; ${ }^{3}$ NUS Graduate School for Integrative Sciences and Engineering, 117456 Singapore; ${ }^{4}$ Laboratory of Cancer Epigenome, Division of Medical Science, National Cancer Centre Singapore, 169610 Singapore; ${ }^{5}$ Lymphoma Genomic Translational Research Laboratory, Division of Medical Oncology, National Cancer Centre Singapore, 169610 Singapore; ${ }^{6}$ Department of Gastroenterology and Metabolism, Graduate School of Biomedical and Health Sciences, Hiroshima University, 1-2-3, Kasumi, Minami-ku, Hiroshima 734-8551, Japan; ${ }^{7}$ Division of Medical Sciences, National Cancer Centre Singapore, 169610 Singapore; ${ }^{8}$ Laboratory for Genome Sequencing Analysis, RIKEN Center for Integrative Medical Sciences, Tokyo 108-8639, Japan

\begin{abstract}
Cisplatin reacts with DNA and thereby likely generates a characteristic pattern of somatic mutations, called a mutational signature. Despite widespread use of cisplatin in cancer treatment and its role in contributing to secondary malignancies, its mutational signature has not been delineated. We hypothesize that cisplatin's mutational signature can serve as a biomarker to identify cisplatin mutagenesis in suspected secondary malignancies. Knowledge of which tissues are at risk of developing cisplatin-induced secondary malignancies could lead to guidelines for noninvasive monitoring for secondary malignancies after cisplatin chemotherapy. We performed whole genome sequencing of 10 independent clones of cisplatin-exposed MCF-10A and HepG2 cells and delineated the patterns of single and dinucleotide mutations in terms of flanking sequence, transcription strand bias, and other characteristics. We used the mSigAct signature presence test and nonnegative matrix factorization to search for cisplatin mutagenesis in hepatocellular carcinomas and esophageal adenocarcinomas. All clones showed highly consistent patterns of single and dinucleotide substitutions. The proportion of dinucleotide substitutions was high: $8.1 \%$ of single nucleotide substitutions were part of dinucleotide substitutions, presumably due to cisplatin's propensity to form intra- and interstrand crosslinks between purine bases in DNA. We identified likely cisplatin exposure in nine hepatocellular carcinomas and three esophageal adenocarcinomas. All hepatocellular carcinomas for which clinical data were available and all esophageal cancers indeed had histories of cisplatin treatment. We experimentally delineated the single and dinucleotide mutational signature of cisplatin. This signature enabled us to detect previous cisplatin exposure in human hepatocellular carcinomas and esophageal adenocarcinomas with high confidence.
\end{abstract}

[Supplemental material is available for this article.]

For 40 years, cisplatin and its derivatives have been cornerstones of the treatment of almost every type of cancer (Dasari and Tchounwou 2014; Dugbartey et al. 2016). However, cisplatin treatment often causes numerous side effects, including hepatotoxicity (Waseem et al. 2015; Dugbartey et al. 2016), and it increases the risk of developing secondary malignancies. For example, cisplatin-based treatments almost always cure testicular cancers but increase the risk of developing a solid tumor later in life 1.8-fold (Travis et al. 2005), and cisplatin treatment of several types of cancers increases the incidence of secondary leukemias (Ratain et al. 1987; Kushner et al. 1998). Cisplatin's therapeutic properties depend partly on its DNA damaging activity, and the risk of secondary malignancies presumably stems from the consequent mutagenesis (Choi et al. 2014). This highlights the importance of understanding cisplatin mutagenesis and how it promotes carci-

Corresponding author: steve.rozen@duke-nus.edu.sg

Article published online before print. Article, supplemental material, and publication date are at http://www.genome.org/cgi/doi/10.1101/gr.230219.117. nogenesis. This also highlights the need for a biomarker to identify cisplatin-induced secondary malignancies.

The mechanisms of cisplatin-induced DNA damage have been extensively studied. When cisplatin enters the cells, its two chloride atoms are hydrolyzed, resulting in two positive charges (Masters and Koberle 2003; Behmand et al. 2015). Although the hydrolyzed molecule presumably reacts with many molecules in the cell, its therapeutic cytotoxicity is generally considered to stem from reactions with the N7 atoms of purine bases in DNA (Harrington et al. 2010; Dasari and Tchounwou 2014; Behmand et al. 2015). Most cisplatin-DNA adducts are crosslinks between two adjacent guanines (GpG, 65\%) or between an adenine and a guanine (5'-ApG-3', 25\%). Mono-adducts and inter-strand crosslinks are much rarer (Jamieson and Lippard 1999; Masters and Koberle 2003; Enoiu et al. 2012). Cisplatin-induced DNA intra-

C 2018 Boot et al. This article is distributed exclusively by Cold Spring Harbor Laboratory Press for the first six months after the full-issue publication date (see http://genome.cshlp.org/site/misc/terms.xhtml). After six months, it is available under a Creative Commons License (Attribution-NonCommercial 4.0 International), as described at http://creativecommons.org/licenses/by-nc/4.0/. 
strand crosslinks and mono-adducts are repaired through nucleotide excision repair (NER) (Zamble et al. 1996; Reardon et al. 1999; Hu et al. 2016). Inter-strand crosslinks are the most difficult to repair and the most cytotoxic because they covalently link the two strands of the DNA helix and consequently block transcription and replication Jamieson and Lippard 1999; Masters and Koberle 2003; Enoiu et al. 2012; Hashimoto et al. 2016; Roy and Scharer 2016). The mechanisms of inter-strand-crosslink repair have not yet been fully elucidated but appear to be complicated (Hashimoto et al. 2016; Roy and Scharer 2016).

Cisplatin likely causes a characteristic pattern of somatic mutations, known as a mutational signature, along with possible additional features such as fewer mutations on the transcribed strands of genes (Alexandrov et al. 2013a). Currently, 30 mutational signatures are widely recognized, and they have a variety of known, suspected, or unknown causes (Alexandrov et al. 2013a,b; Forbes et al. 2017; cancer.sanger.ac.uk). Mutational signatures can serve as biomarkers for endogenous mutagenic processes and exogenous exposures that lead to the development of tumors.

We hypothesize that cisplatin's mutational signature can serve as a biomarker to identify cisplatin mutagenesis in suspected secondary malignancies. Knowledge of which tissues are at risk of developing cisplatin-induced secondary malignancies could lead to guidelines for noninvasive monitoring for secondary malignancies after cisplatin chemotherapy.

Two previous studies investigated the mutational signature of cisplatin, one in Caenorhabditis elegans and one in a chicken (Gallus gallus) B-cell cell line (Meier et al. 2014; Szikriszt et al. 2016). Although both studies reported mutational signatures with primarily $\mathrm{C}>\mathrm{A}$ mutations, the single-nucleotide substitution (SNS) signatures were otherwise dissimilar: The C. elegans signature was dominated by CㅡA $>$ CAAA and CECT $>$ CAT mutations, while the chicken signature was dominated by $\overline{\mathrm{C} C}>\mathrm{AC}$ mutations. This lack of similarity may have been due to the different model systems used, to the low numbers of mutations in the $C$. elegans study, or to experimental differences between the studies. In any case, these studies failed to unequivocally elucidate the mutational signature of cisplatin.

Therefore, we studied cisplatin mutations in MCF-10A, a nontumorigenic human breast epithelial cell line, and in HepG2, a human liver cancer cell line. Here, we report the extensive characterization of the cisplatin signature obtained, as well as its discovery in hepatocellular carcinomas and esophageal adenocarcinomas in patients previously exposed to cisplatin.

\section{Results}

\section{Cisplatin's single-nucleotide substitution signature}

We exposed two independent cultures of MCF-10A cells to 0.5 and $1 \mu \mathrm{M}$ of cisplatin and one culture of HepG2 to $0.75 \mu \mathrm{M}$ of cisplatin once a week for $8 \mathrm{wk}$. Single cells were isolated and expanded for whole-genome sequencing and mutational analysis. We sequenced the untreated cell lines, three MCF-10A clones for each concentration (one exposed for $4 \mathrm{wk}$ and two exposed for $8 \mathrm{wk}$ ) and 4 HepG2 clones (exposed for $8 \mathrm{wk}$ ). Mean coverage was $>33 \times$, and in total we identified 70,313 single-nucleotide substitutions (Supplemental Table S1).

The SNS mutation spectra from all clones were highly similar (all Pearson correlations $>0.958$ and cosine similarities $>0.971$ ) (Fig. 1A; Supplemental Fig. S1A; Supplemental Table S2). The most prominent features were two $\mathrm{C}>\mathrm{T}$ peaks $(\mathrm{CCC}>\mathrm{CTC}$ and CㅡT $>$ CTT $)$ and four $\mathrm{T}>\mathrm{A}$ peaks $(\mathrm{CT}>\mathrm{CA})$. There were also substantial numbers of $\mathrm{C}>\mathrm{A}$ mutations $(\sim 26.0 \%$ of all mutations) and peaks at GCC $>$ GAC and GCC $>$ GGC. Figure $1 \mathrm{~B}$ and Supplemental Figure S1B display the signatures as mutation rates per trinucleotide, which better reflects the sequence specificity of mutational processes because they are not affected by differences in trinucleotide abundances. For example, Figure 1B shows more prominent CCC > CTC peaks and reveals that the gap at CCG > CTG in Figure $1 \mathrm{~A}$ reflects the low abundance of CCG trinucleotides in the genome rather than reduced mutagenicity.

In addition to consistent patterns of the bases immediately $5^{\prime}$ and $3^{\prime}$ of cisplatin SNSs, there were also many preferences $2 \mathrm{bp}$ $5^{\prime}$ and $3^{\prime}$ of the SNSs (Fig. 1C; Supplemental Fig. S2). For example, $\mathrm{CT}>\mathrm{CA}$ mutations were usually preceded by an $\mathrm{A}$ (ACT $>\mathrm{ACA})$. Similarly, CC $>C \underline{C}$ mutations were usually preceded by a pyrimidine (YCC $>$ YCT). These and other preferences at the $-2 \mathrm{bp}$ or +2 bp positions were statistically significant (Supplemental Fig. S3). Examination of the -3 bp or +3 bp positions of SNSs revealed no additional sequence context preferences (Supplemental Fig. S4).

\section{Associations of cisplatin-induced single-nucleotide substitutions with genomic features}

Many mutational processes cause fewer mutations due to damage on the transcribed strands of genes than on the nontranscribed strands. This is termed transcription strand bias and is due to transcription-coupled nucleotide excision repair (TC-NER) of adducted bases in the transcribed (antisense) strands. Since cisplatin forms adducts on purines, we would expect reduced numbers of mutations when $\mathrm{G}$ and $\mathrm{A}$ are on the transcribed strand (corresponding to $\mathrm{C}$ and $\mathrm{T}$ on the sense strand). As expected, $\mathrm{C}>\mathrm{A}, \mathrm{C}>\mathrm{T}$, and $\mathrm{T}>\mathrm{A}$ SNSs were strongly reduced on the sense strand (Supplemental Fig. S5; Fousteri and Mullenders 2008; Harrington et al. 2010; Dasari and Tchounwou 2014; Behmand et al. 2015; $\mathrm{Hu}$ et al. 2016). Also consistent with TC-NER, strand bias for $\mathrm{C}>\mathrm{A}, \mathrm{C}>\mathrm{T}$, and $\mathrm{T}>\mathrm{A}$ mutations was stronger in more highly expressed genes $\left(P=1.45 \times 10^{-50}\right.$ and $1.20 \times 10^{-116}$, one-sided $\chi^{2}$ test for all MCF$10 \mathrm{~A}$ and for all HepG2 clones combined, respectively) (Fig. 2A; Supplemental Fig. S6). Finally, TC-NER efficiency decreases from the $5^{\prime}$ to the $3^{\prime}$ ends of transcripts (Conaway and Conaway 1999; $\mathrm{Hu}$ et al. 2015; Huang et al. 2017). Consistent with this, strand bias for $\mathrm{C}>\mathrm{A}, \mathrm{C}>\mathrm{T}$, and $\mathrm{T}>\mathrm{A}$ SNSs decreased toward the $3^{\prime}$ ends of transcripts $\left(P=2.46 \times 10^{-12}\right.$ and $P=1.85 \times 10^{-12}$, logistic regression for all MCF-10A clones and for all HepG2 clones combined, respectively) (Fig. 2B; Supplemental Fig. S7).

In addition to transcription strand bias, cisplatin mutagenesis also showed replication timing bias, with a higher mutation density in late replicating regions $\left(P=9.39 \times 10^{-73}\right.$ and $1.96 \times 10^{-136}$, binomial test for MCF-10A and HepG2 clones, respectively). We noted high variability in replication timing bias between the different clones, the cause of which remains unclear. Interestingly, $\mathrm{C}>\mathrm{T}$ mutations showed lower replication timing bias than other mutations classes (Supplemental Fig. S8). There was no difference in mutation density between leading and lagging replication strands.

For some mutational processes, mutagenesis intensity varies by chromatin state (Polak et al. 2015; Seplyarskiy et al. 2015; Kaiser et al. 2016). Additionally, there is increased cisplatin adduct formation in open chromatin compared to closed chromatin ( $\mathrm{Hu}$ et al. 2016). In both cell lines, regions containing active promoters, enhancers, and actively transcribed genes were less highly 
Boot et al.
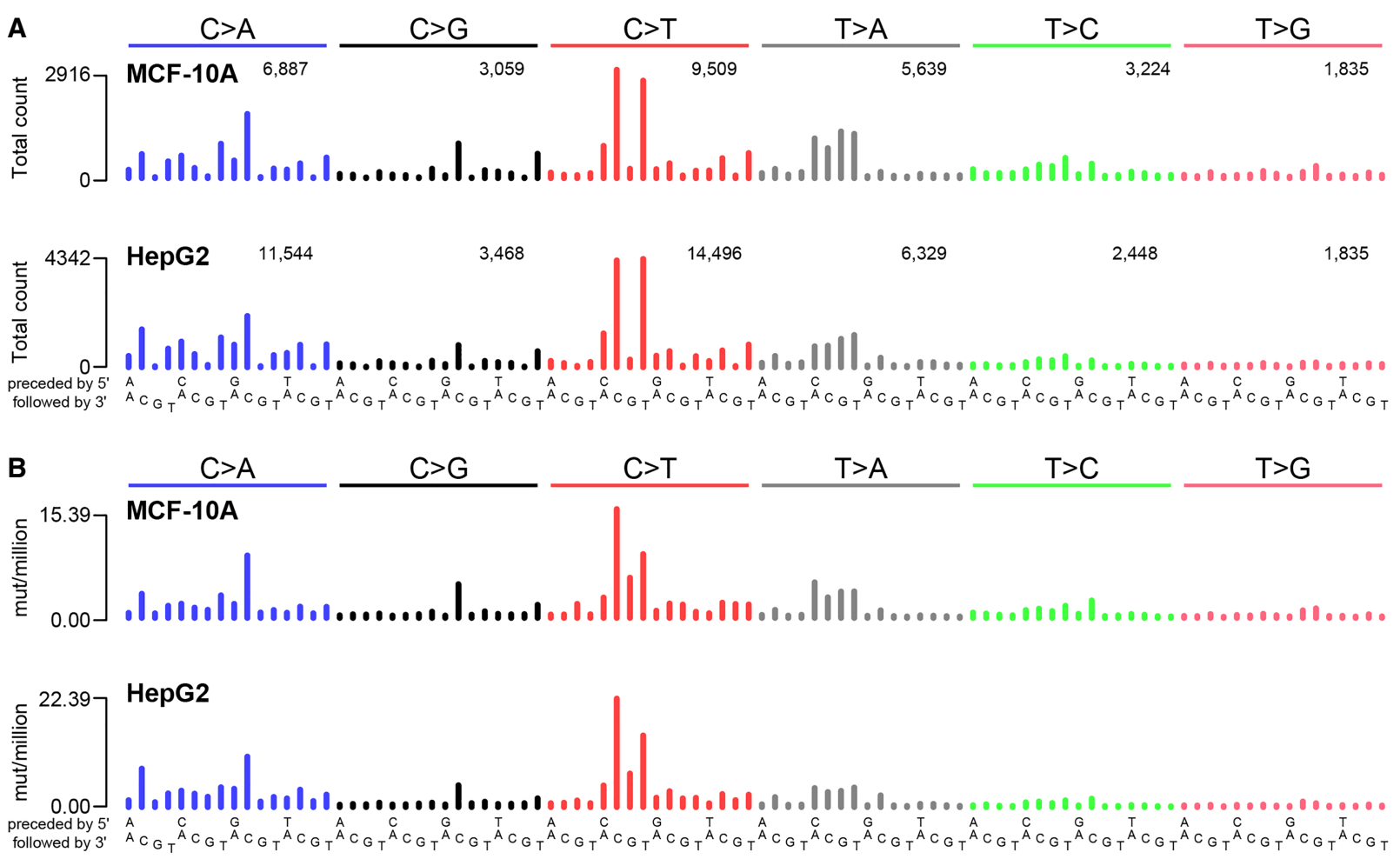

C
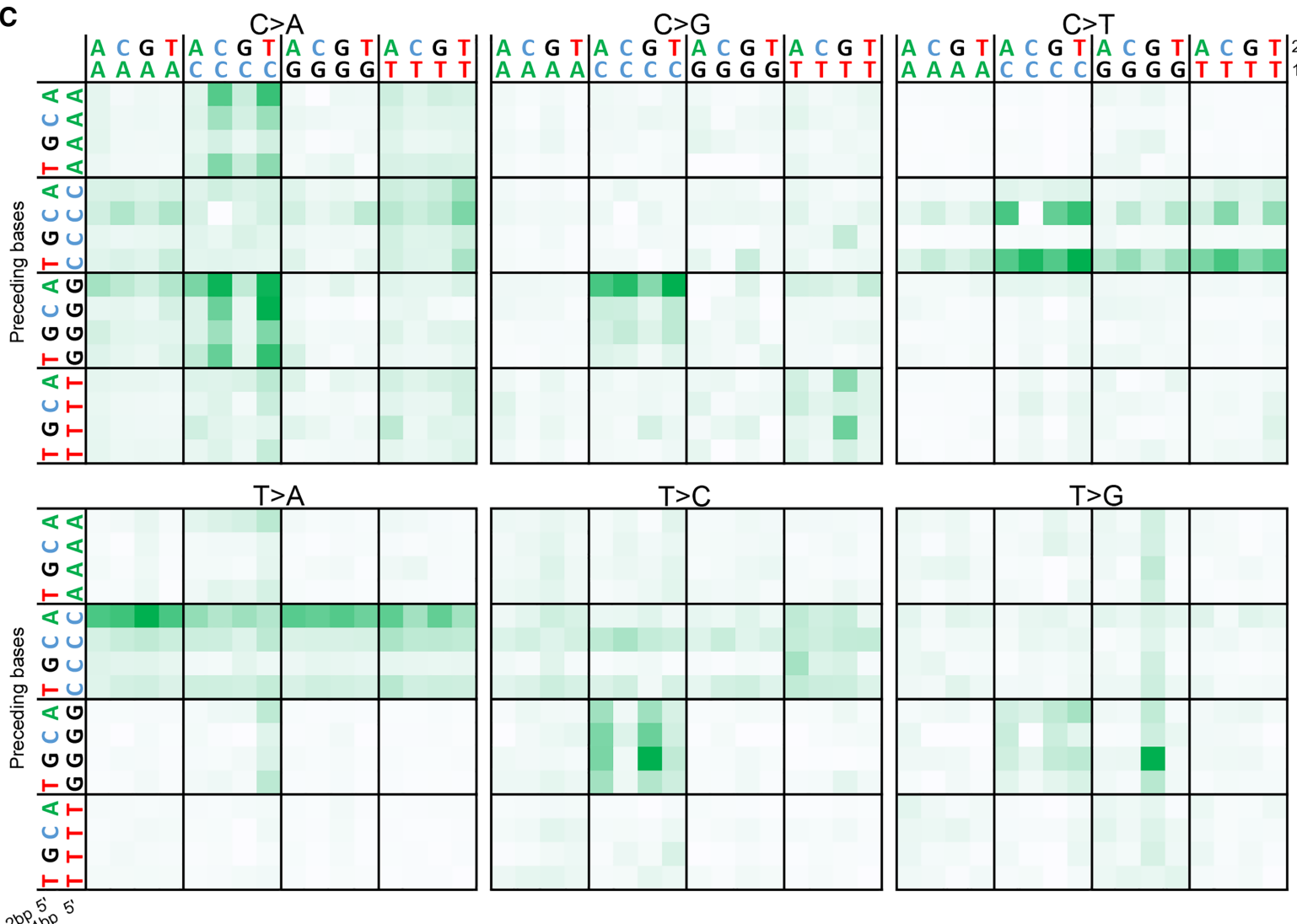

Figure 1. Cisplatin mutational signature. Trinucleotide-context mutational spectra shown as $(A)$ raw counts and $(B)$ rate of mutations per million trinucleotides for all MCF-10A (top panel) and all HepG2 (bottom panel) clones combined. In $A$, the number of mutations per SNS type is shown above the corresponding bars. (C) Pentanucleotide sequence contexts for all samples combined, normalized by pentanucleotide occurrence in the genome. See also Supplemental Figure S2. 
A
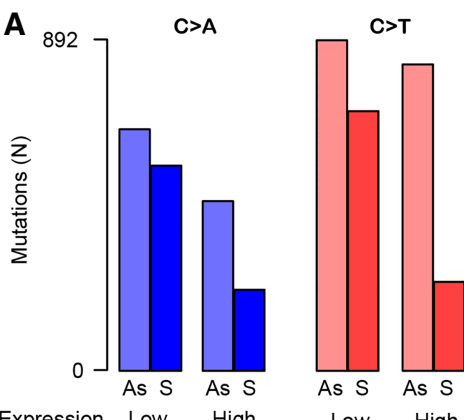

C

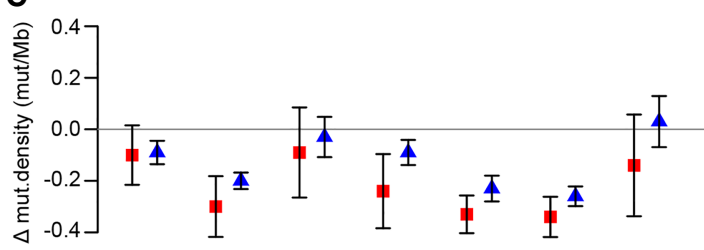

B
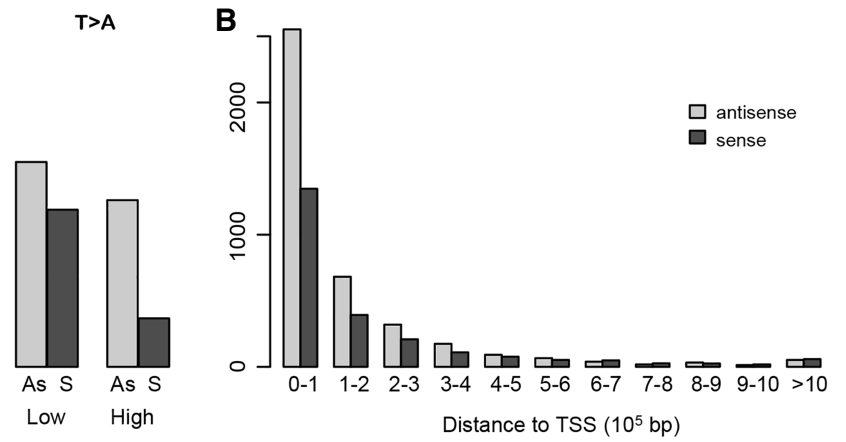

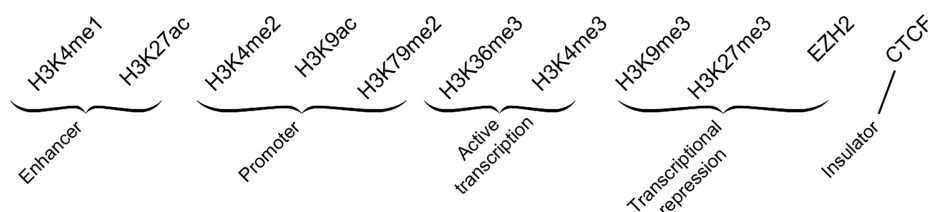

Figure 2. Associations between cisplatin mutagenesis intensity and genomic features. (A) Transcription strand bias is more prominent in highly expressed genes for C >A, C > T, and T > A mutations. See also Supplemental Figure S6. (B) Transcription strand bias decreases with increasing distance from the transcription start site (TSS). See also Supplemental Figure S7. Mutations were binned per 100,000 bp, i.e., the first bars are the numbers of mutations within the first 100,000 bp from the TSS, the next bars are the numbers of mutations in the region from 100,001 to 200,000 bp from the TSS, and so on. (C) Mutation density in regions with histone modifications and in binding sites for EZH2 and CTCF. The $y$-axis is the mean mutation density for the given region relative to the mutation density of each respective sample; bars show standard error of the mean (Supplemental Table S1).

mutated, and regions associated with heterochromatin and transcriptional repression were more highly mutated (Fig. 2C).

\section{Cisplatin's dinucleotide substitution signature}

To investigate the presence of dinucleotide substitutions (DNSs) in the cisplatin genomes, we selected all adjacent SNSs and verified that both SNSs were on the same reads (see Methods). We identified 2839 DNSs in the cisplatin genomes, of which most were mutations from CC, CT, TC, and TG (Fig. 3A; Supplemental Fig. S9). We hypothesized that mutations from CC, CT, and TC are consequences of intra-strand crosslinks at $\mathrm{GpG}, \mathrm{ApG}$, and $\mathrm{GpA}$, and that mutations from TG were consequences of diagonally offset interstrand guanine-adenine crosslinks ${ }_{3^{\prime}}^{{ }^{\prime} \mathbf{T C}^{\mathbf{A}^{\prime}} \mathbf{C}_{5^{\prime}}}$ (crosslinked bases in bold). Mutations from AT, TA, and TT were rare, which is consistent with previous reports that cisplatin does not induce adenine-adenine crosslinks (Supplemental Table S3; Jamieson and Lippard 1999; Masters and Koberle 2003).

The proportion of SNSs involved in DNSs ranged from $6.2 \%$ to $9.2 \%$. To relate this to other mutagenic processes known to be associated with DNSs, we examined the percentage of SNSs involved in DNSs associated with COSMIC Signatures 4 (smoking-related) and 7 (due to UV exposure) (Forbes et al. 2017; cancer.sanger. ac.uk). We studied Signature 4 in 24 lung adenocarcinomas (Imielinski et al. 2012) and Signature 7 in 112 melanomas (Zhang et al. 2011). In both tumor types, the percentage of SNSs involved in DNSs was significantly lower than in cisplatin (mean
$3.5 \%, \mathrm{SD}=1.4 \%, P=6.5 \times 10^{-10}$ and mean $=3.3 \%, \mathrm{SD}=1.6 \%, P=$ $4.6 \times 10^{-14}$, respectively, two-sided $t$-tests versus cisplatin) (Fig. 3B). We hypothesize that this high proportion of DNSs in cisplatin stems from cisplatin's propensity to form intra-strand crosslinks between adjacent bases and to form diagonally offset inter-strand crosslinks.

To investigate possible sequence context preferences of cisplatin DNSs, we plotted 1-bp contexts of each reference dinucleotide, irrespective of the mutant allele (Fig. 3C; Supplemental Fig. S10). There was strong enrichment for TC and TG DNSs in TCT and TGG contexts. Both TC and TG DNSs were further enriched for a $\overline{5^{\prime}}$ flanking purine (Supplemental Figs. S10, S11). The strongest sequence context preference was for CC $>\mathrm{NN}$ mutations, $49.8 \%$ of which occurred in the GCCT context (Supplemental Figs. S10, S11). As methodological controls, we also evaluated \pm 1 -bp sequence context for DNSs associated with COSMIC Signatures 4 and 7. DNSs associated with Signature 7 showed strong sequence context preference for most mutation classes, including $\mathrm{CC}>\mathrm{NN}$, $\mathrm{CT}>\mathrm{NN}$, and TT $>$ NN (Supplemental Fig. S12). The context preferences were very different, however, from those of cisplatin DNSs. In contrast, DNSs associated with Signature 4 had only weak sequence context preferences (Supplemental Fig. S12).

\section{Associations of cisplatin-induced dinucleotide substitutions with genomic features}

To assess transcription strand bias in DNSs, we examined separately the mutations hypothetically involving inter-strand 

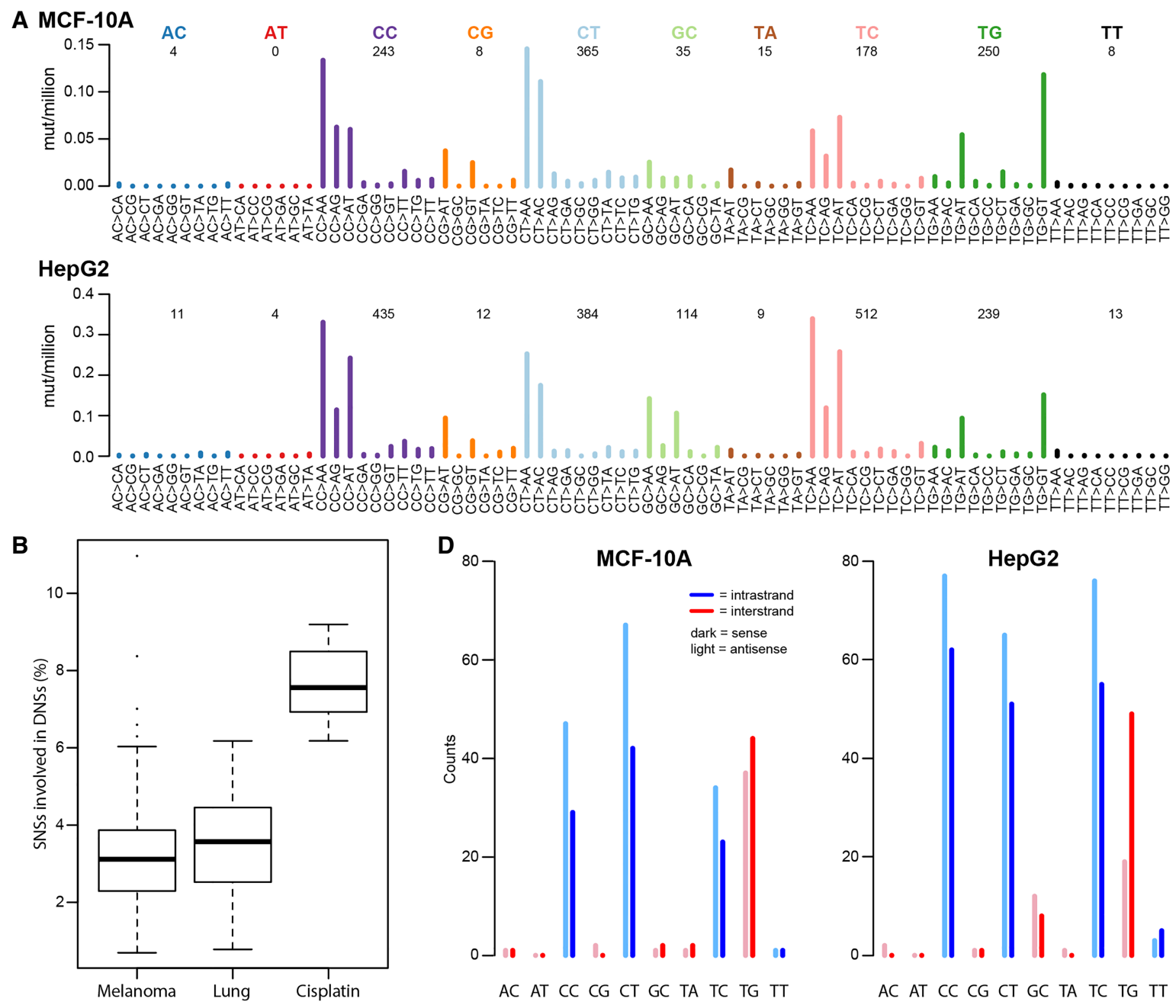

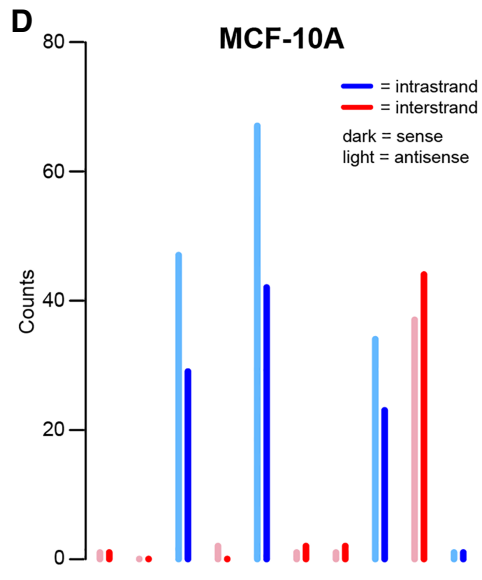

AC AT CC CG CT GC TA TC TG TT

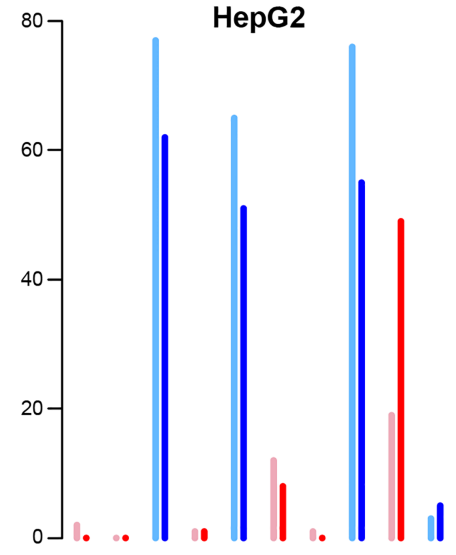

AC AT CC CG CT GC TA TC TG TT
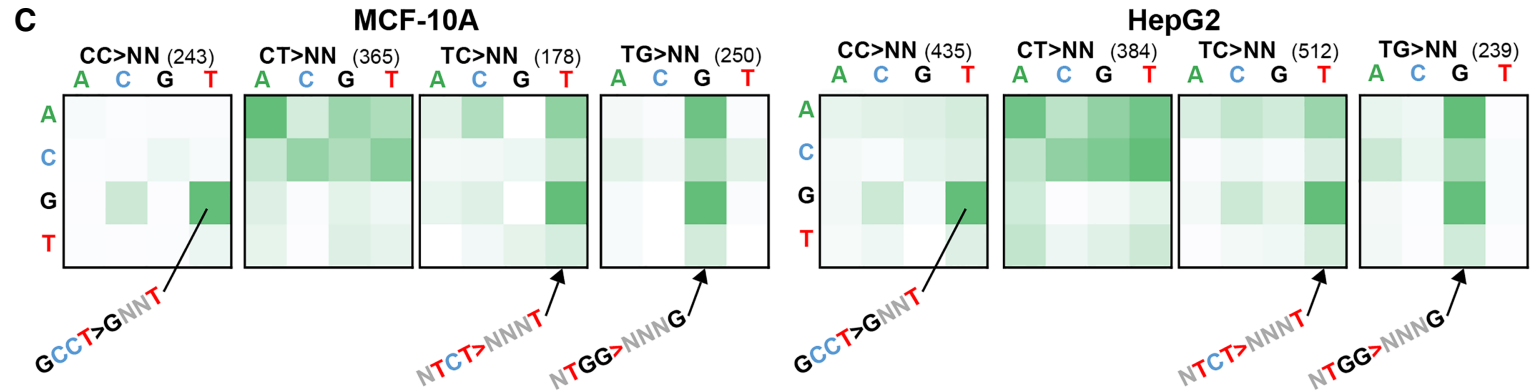

Figure 3. Cisplatin-induced dinucleotide substitutions (DNSs). ( $A$ ) DNS mutation spectra of all MCF-10A (top panel) and all HepG2 (bottom panel) clones combined, displayed as DNSs per million dinucleotides (i.e., normalized for dinucleotide abundance in the genome). ( $B$ ) Cisplatin induces higher numbers of DNSs than other mutational processes associated with dinucleotide substitutions such as UV (melanoma) and smoking (lung). (C) \pm 1 -bp sequence context preferences for the most prominent DNS mutation classes (CC $>N N, C T>N N, T C>N N$, and TG $>N N$ ). The total number of DNSs per mutation class is indicated in parentheses. The vertical axis is the preceding (5') base, the horizontal axis is the following ( $\left.3^{\prime}\right)$ base. Some prominent enrichments in sequence context are indicated (GCCT > GNNT, NTCT > NNNT, and NTGG > NNNG). The full sequence context preference plots, both raw counts and normalized for tetranucleotide abundance in the genome, are shown in Supplemental Figure S10. (D) Transcription strand bias of dinucleotide substitutions. Potential intra-strand crosslink sites are shown in blue, potential inter-strand crosslink sites are shown in red.

purine-purine crosslinks, predominantly mutations from the ${ }^{5^{\prime}} \mathbf{T G}^{3^{\prime}}$ ${ }_{3} \mathbf{A C}_{5^{\prime}}$ configuration, and the mutations hypothetically involving intra-strand purine-purine crosslinks (predominantly mutations from CC, CT, and TC). We observed transcription strand bias at the potential intra-strand crosslink sites other than TC in most of the MCF-10A and HepG2 clones (Fig. 3D; Supplemental Fig. S13). There was no consistent evidence of transcription strand bias at potential inter-strand crosslink sites (mainly TG) in the MCF-10A clones. However, for three of the four HepG2 clones, there were fewer mutations when TG was on the transcribed (antisense) strand (Supplemental Fig. S13). As methodological controls,

\section{Genome Research}

www.genome.org 
we also evaluated transcription strand bias for DNSs associated with COSMIC Signatures 4 and 7, in which we also detected strand bias (Supplemental Fig. S14).

With respect to other genomic features, the replication-timing bias of DNSs was similar to that of the SNSs (Supplemental Fig. S8). Association of DNS density with marks of active and repressed chromatin was similar to that of SNS density, with the following exceptions (Supplemental Fig. S15). DNS density was markedly higher than SNS density in regions of H3K9 acetylation and markedly lower at binding sites of EZH2 (enhancer of zeste homolog 2 polycomb repressive complex 2 subunit). In addition, DNS density was markedly higher than SNS density at binding sites of CTCF (CCCTC-binding factor).

\section{Other mutation types}

We also examined small insertion and deletion mutations (indels), copy number alterations, and structural variants in the cisplatinexposed MCF-10A and HepG2 clones. We identified 4208 indels in the cisplatin-exposed clones. The indels were unremarkable, consisting primarily of single-nucleotide insertions or deletions ( 78\%) (Supplemental Fig. S16). Like SNSs, indels were enriched in late replicating regions (Supplemental Fig. S8). The distribution of indels with respect to other genomic features was very similar to that of DNSs (Supplemental Fig. S15). There were very few copy number alterations or structural variants (Supplemental Figs. S17, S18), suggesting that cisplatin did not induce detectable genomic instability.

\section{Likely cisplatin mutational signature in human tumors}

We examined publicly available human tumor mutation data for evidence of the experimental cisplatin signature. Notably, mutational signature W6, which was reported in the whole-genome sequences of hepatocellular carcinomas (HCCs), resembles the experimental cisplatin signature (cosine similarity $=0.781$ ) (Supplemental Fig. S19; Fujimoto et al. 2016). Although the relative proportions of the major substitution classes $(\mathrm{C}>\mathrm{A}, \mathrm{C}>\mathrm{T}$, and $\mathrm{T}>\mathrm{A})$ are rather different between Signature W6 and our experimental cisplatin signature, the profiles within each mutation class are similar (cosine similarities for C > A, C > T, and T > A of 0.915, 0.917, and 0.981, respectively) (Supplemental Fig. S19). Given this resemblance, we searched for the cisplatin SNS signature using the mSigAct signature presence test (see Methods; Ng et al. 2017) in data from Japanese and Hong Kong HCCs (Kan et al. 2013; Fujimoto et al. 2016). Out of 342 HCCs, 10 showed evidence of cisplatin exposure (Table 1; Fig. 4A; Supplemental Fig. S20, cf. Fig. 1A). To further assess presence of cisplatin mutagenesis, we also examined the dinucleotide spectra of these samples (Fig. 4B; Supplemental Fig. S21; cf. Fig. 3A). Seven of the 10 HCCs with the cisplatin SNS spectrum also had high cosine similarities between their DNS spectra and the cisplatin signature (Fig. 4C) and high numbers of DNSs relative to their total SNS load (ranging from $2.9 \%$ to $6.2 \%$, compared to the median of $1.6 \%$, for all HCCs) (Supplemental Fig. S22A).

We also analyzed the mutational spectra of 140 esophageal adenocarcinomas (ESADs), of which 68 had been treated with cisplatin prior to surgery (Noorani et al. 2017). SNS analysis suggested that three of the cisplatin-treated ESADs had the cisplatin signature, whereas we found no evidence of cisplatin mutagenesis in any of the untreated ESADs. The DNS analysis supported likely cisplatin exposure in all ESADs identified in the SNS analysis (Table 1; Supplemental Figs. S22B, S23, S24).
We further investigated whether DNS analysis could identify cisplatin-exposed tumors that were missed by the SNS analysis. We performed semi-supervised Nonnegative Matrix Factorization (ssNMF) on all tumors with $\geq 25$ DNSs, specifying the cisplatin DNS signature as one input signature and asking for discovery of one to four additional signatures (Methods; Supplemental Figs. S25, S26). All seven previously identified cisplatin-positive HCCs had $>50 \%$ DNS attributed to cisplatin by ssNMF, as did an additional 13 HCCs. Among these 13 HCCs, two, RK072 and RK140, had high cosine similarities with the experimental cisplatin DNS signature and had relatively high proportions of DNSs compared to SNSs (Table 1; Supplemental Fig. S22A). Although the SNS-based $P$ values were not significant after multiple-testing correction, we nevertheless concluded, based on the combined SNS and DNS analyses, that RK072 and RK140 showed strong evidence for cisplatin mutagenesis. For the remaining 11 HCCs with $>50 \%$ cisplatin-associated DNSs, neither mSigAct nor visual inspection of the SNS spectra warranted reclassification as cisplatin-positive.

Use of ssNMF also identified high proportions of cisplatinassociated DNSs in several ESADs. These included the three identified in our initial analysis. Of the remainder, neither the mSigAct signature presence test on the SNSs nor visual inspection of the SNS spectra warranted reclassification as cisplatin-positive. None of the chemotherapy naive ESADs displayed signs of cisplatin mutagenesis based on the DNS analysis.

Beyond the mutation frequency spectrum, the other characteristics of the DNSs in the cisplatin-positive HCCs and ESADs were very similar to DNS characteristics in the experimental data. First, the DNS sequence context preference of the cisplatin-positive HCCs and ESADs was extremely similar to the experimental data (Fig. 4D, cf. Fig. 3C). The TC and TG DNSs were less frequent in the tumors than in the experimental data but nevertheless showed very similar sequence context preferences. Second, like the cisplatin-exposed cells, most HCCs and ESADs showed strong transcription strand bias at CC and CT DNSs but not at TC DNSs (Fig. 4E; Supplemental Fig. S27). Also like the cisplatin-exposed MCF-10A clones, none of the HCCs and ESADs had detectable transcription strand bias at potential inter-strand crosslink sites (mainly TG DNSs) (Supplemental Fig. S27). Third, like the experimental data, the DNSs of the cisplatin-positive tumors did not show replication strand bias but did show strong replication timing bias (Fig. 4F).

The clinical records of the Japanese HCCs (Fujimoto et al. 2016) confirmed cisplatin exposure of all of the eight HCCs identified positive for the cisplatin mutational signature (Table 1). All seven had received cisplatin-based trans-arterial chemoembolization using drug eluting beads (DEB-TACE) several months prior to surgical resection. In addition to DEB-TACE treatment of the sampled tumor, patients RK205, RK241, and RK256 also had prior malignancies (Table 1). The variant allele frequencies of the cisplatin-associated DNSs were similar to the variant allele frequencies of all SNSs, including those not likely due to cisplatin exposure (Supplemental Table S4). This suggested that the cisplatin was an early event in tumorigenesis, which would be concordant with rapid clonal expansion after DEB-TACE treatment (Zen et al. 2011). Notably, the three HCCs (RK047, RK223, and RK309) that we suspected to be false-positives based on DNS analysis had no record of treatment with cisplatin prior to surgery.

\section{Discussion}

We have delineated the in vitro multidimensional mutational signature of cisplatin in two human cell lines. This comprised 
Table 1. HCCs and ESADs with cisplatin-associated mutagenesis

\begin{tabular}{|c|c|c|c|c|c|c|c|c|c|c|}
\hline Tumor ID & $\begin{array}{l}\text { Cancer } \\
\text { type }\end{array}$ & $\begin{array}{l}\text { Total } \\
\text { SNSs }\end{array}$ & $\begin{array}{l}\text { Cisplatin } \\
\text { SNSs }\end{array}$ & $\underset{\mathbf{P}^{\mathbf{a}}}{\mathrm{mSigAct}}$ & DNSs & $\begin{array}{l}\text { DNS cosine } \\
\text { similarity to } \\
\text { experimental } \\
\text { signature }\end{array}$ & $\begin{array}{l}\text { DNSs due } \\
\text { to } \\
\text { cisplatin }^{\text {b }}\end{array}$ & $\begin{array}{c}\text { Conclusion based } \\
\text { on SNS and DNS } \\
\text { analysis }\end{array}$ & Patient history ${ }^{c}$ & Reference \\
\hline НK034 & $\mathrm{HCC}$ & 7844 & 2274 & $1.3 \times 10^{-11}$ & 153 & 0.827 & 130 & Cisplatin-positive & NA & $\begin{array}{c}\text { Kan et al. } \\
2013\end{array}$ \\
\hline RK028 & $\mathrm{HCC}$ & 20,792 & 7594 & $1.5 \times 10^{-21}$ & 642 & 0.773 & 533 & Cisplatin-positive & Cisplatin DEB-TACE & $\begin{array}{l}\text { Fujimoto } \\
\text { et al. } \\
2016\end{array}$ \\
\hline RK047 & $\mathrm{HCC}$ & 8345 & 1522 & $1.1 \times 10^{-4}$ & 73 & 0.498 & 8 & Negative & $\begin{array}{r}\text { No neo-adjuvant } \\
\text { chemotherapy }\end{array}$ & $\begin{array}{l}\text { Fujimoto } \\
\text { et al. } \\
2016\end{array}$ \\
\hline RK056 & $\mathrm{HCC}$ & 17,085 & 7097 & $4.4 \times 10^{-26}$ & 479 & 0.874 & 426 & Cisplatin-positive & Cisplatin DEB-TACE & $\begin{array}{l}\text { Fujimoto } \\
\text { et al. } \\
2016\end{array}$ \\
\hline RK072 & $\mathrm{HCC}$ & 8893 & 1130 & $4.9 \times 10^{-4}$ & 92 & 0.698 & 50 & Cisplatin-positive & $\begin{array}{l}\text { Cisplatin DEB-TACE } \\
\text { (five rounds) }\end{array}$ & $\begin{array}{l}\text { Fujimoto } \\
\text { et al. } \\
2016\end{array}$ \\
\hline RK074 & $\mathrm{HCC}$ & 22,406 & 6903 & $2.5 \times 10^{-18}$ & 476 & 0.865 & 415 & Cisplatin-positive & Cisplatin DEB-TACE & $\begin{array}{l}\text { Fujimoto } \\
\text { et al. } \\
2016\end{array}$ \\
\hline RK140 & $\mathrm{HCC}$ & 10,132 & 1115 & $2.5 \times 10^{-4}$ & 125 & 0.800 & 73 & Cisplatin-positive & $\begin{array}{l}\text { Cisplatin DEB-TACE, } \\
4 \mathrm{yr}, 1 \mathrm{yr} \text {, and } \\
6 \mathrm{mo} \text { prior }\end{array}$ & $\begin{array}{l}\text { Fujimoto } \\
\text { et al. } \\
2016\end{array}$ \\
\hline RK205 & $\mathrm{HCC}$ & 10,406 & 1751 & $3.0 \times 10^{-6}$ & 158 & 0.720 & 150 & Cisplatin-positive & $\begin{array}{l}\text { Cisplatin DEB-TACE, } \\
\text { prior history of } \\
\text { HCC, resected } \\
27 \text { mo ago }\end{array}$ & $\begin{array}{l}\text { Fujimoto } \\
\text { et al. } \\
2016\end{array}$ \\
\hline RK223 & $\mathrm{HCC}$ & 10,680 & 1157 & $1.4 \times 10^{-4}$ & 141 & 0.640 & 65 & Negative & $\begin{array}{l}\text { No neo-adjuvant } \\
\text { chemotherapy }\end{array}$ & $\begin{array}{l}\text { Fujimoto } \\
\text { et al. } \\
2016\end{array}$ \\
\hline RK241 & $\mathrm{HCC}$ & 10,610 & 3373 & $5.1 \times 10^{-16}$ & 235 & 0.767 & 177 & Cisplatin-positive & $\begin{array}{l}\text { Cisplatin DEB-TACE, } \\
\text { prior history of } \\
\text { colorectal cancer }\end{array}$ & $\begin{array}{l}\text { Fujimoto } \\
\text { et al. } \\
2016\end{array}$ \\
\hline RK256 & $\mathrm{HCC}$ & 11,240 & 2530 & $2.4 \times 10^{-9}$ & 167 & 0.806 & 142 & Cisplatin-positive & $\begin{array}{l}\text { Cisplatin DEB-TACE, } \\
\text { prior history of } \\
\text { HCC, resected } 37 \\
\text { and } 18 \text { mo ago }\end{array}$ & $\begin{array}{l}\text { Fujimoto } \\
\text { et al. } \\
2016\end{array}$ \\
\hline RK309 & $\mathrm{HCC}$ & 4785 & 1379 & $2.3 \times 10^{-5}$ & 12 & 0.558 & $\begin{array}{l}\text { Too few } \\
\text { DNSs to } \\
\text { analyze }\end{array}$ & Negative & $\begin{array}{l}\text { No neo-adjuvant } \\
\text { chemotherapy }\end{array}$ & $\begin{array}{l}\text { Fujimoto } \\
\text { et al. } \\
2016\end{array}$ \\
\hline SA594320 & ESAD & 23,483 & 6288 & $3.9 \times 10^{-17}$ & 313 & 0.902 & 266 & Cisplatin-positive & Cisplatin-treated & $\begin{array}{l}\text { Noorani } \\
\text { et al. } \\
2017\end{array}$ \\
\hline SA594557 & ESAD & 7433 & 780 & $3.0 \times 10^{-4}$ & 63 & 0.877 & 41 & Cisplatin-positive & Cisplatin-treated & $\begin{array}{c}\text { Noorani } \\
\text { et al. } \\
2017\end{array}$ \\
\hline SA594775 & ESAD & 14,967 & 2147 & $3.0 \times 10^{-5}$ & 124 & 0.790 & 58 & Cisplatin-positive & Cisplatin-treated & $\begin{array}{l}\text { Noorani } \\
\text { et al. } \\
2017\end{array}$ \\
\hline
\end{tabular}

aBonferroni level of significance is $1.5 \times 10^{-4}(0.05 / 342)$ for the HCCs and $3.6 \times 10^{-4}(0.05 / 140)$ for the ESADs.

${ }^{b}$ DNS assignment by ssNMF. The cisplatin DNS signature was given as input, and two other DNS signatures were requested. Reported here is the number of DNSs assigned to the cisplatin DNS signature.

${ }^{\mathrm{C}} \mathrm{NA}$ denotes data not available.

extensive characterization of patterns of SNSs in tri- and pentanucleotide contexts and the associations of SNSs with genomic features. We also found patterns of DNSs and flanking bases and the associations of DNSs with genomic features that were highly informative. We began with in vitro delineation because it directly links mutational signatures to etiologies and because it generates signatures that are relatively unobscured by other mutational processes. We expect a small number of mutations due to clock-like mutational processes (signatures 1 and 5) to be present in the experimental cisplatin signature (Alexandrov et al. 2015). We propose that these do not obscure the important characteristics of the experimental cisplatin signatures, because this signature does not even remotely resemble any other in vitro mutational signa- ture (Poon et al. 2013; Olivier et al. 2014; Huang et al. 2017; Zhivagui et al. 2018). If the experimental cisplatin signature reported here were due primarily to a background mutational process, it would have to be a process that was never previously observed in vitro and that arose independently and almost identically in two different cell lines. Therefore, it is extremely unlikely that the experimental cisplatin mutational signature is the result of a background mutational process.

We analyzed whole-genome data because these provide $>50$ times more mutations than exomes and consequently greater stability and reproducibility of signatures. Indeed, whole-genome data are practically essential for analysis of DNSs, which are rare compared to SNSs. Importantly, with the experimentally

\section{Genome Research}

www.genome.org 
A

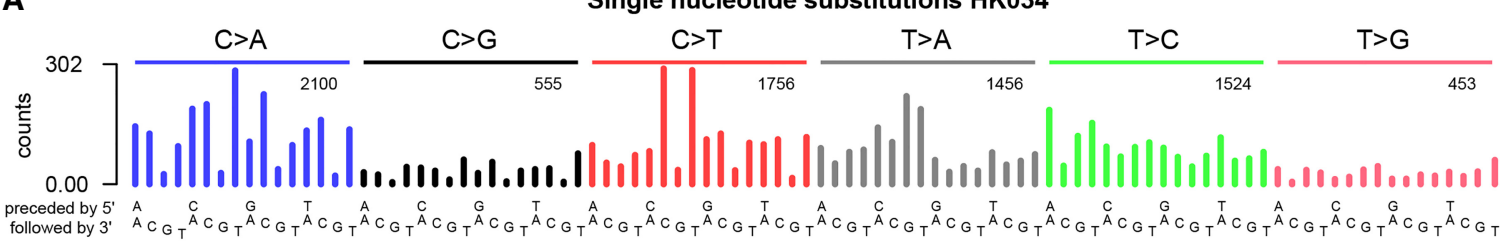

B

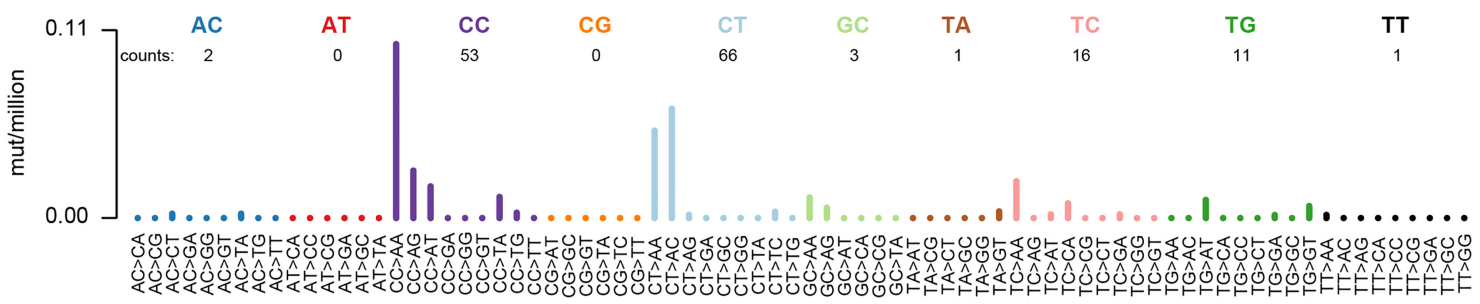

C

Similarity of in vitro data and HCC dinucleotide substitutions

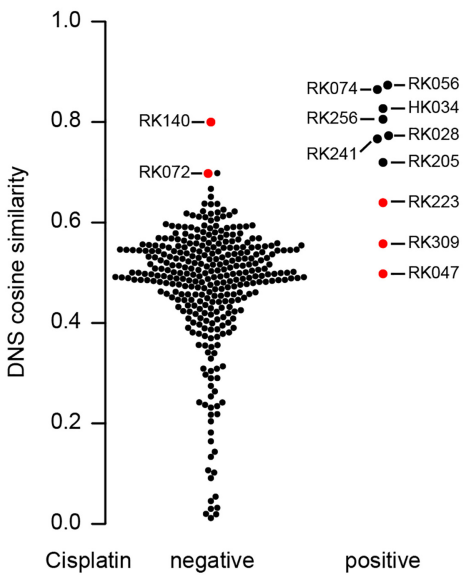

(by SNS analysis)
E

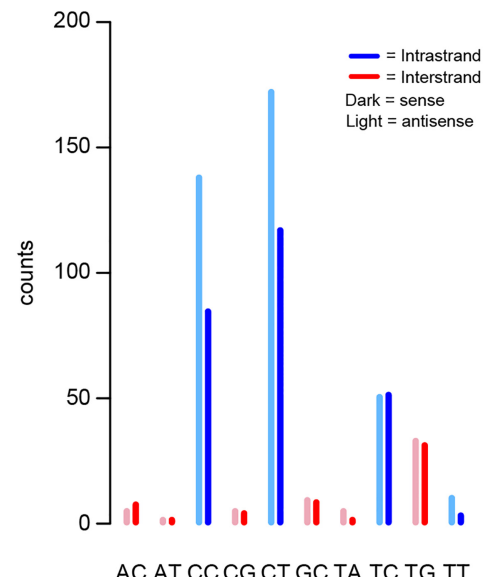

$\mathbf{F}$

DNS replication timing bias for all cisplatin positive HCCs

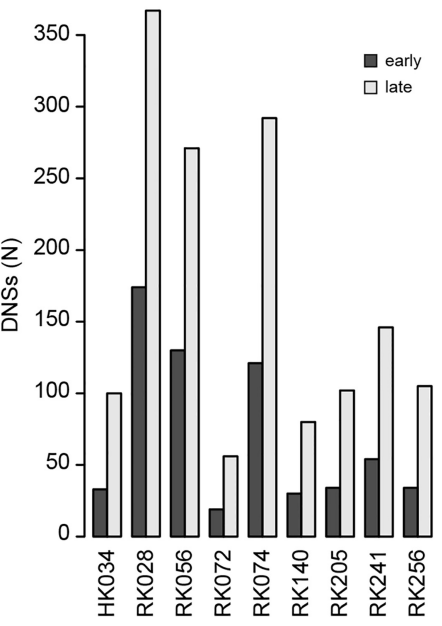

D

HCCs
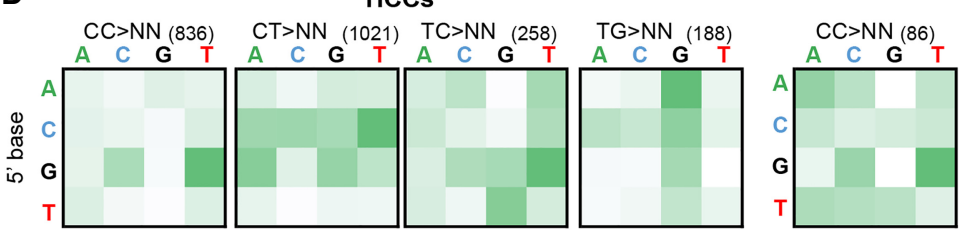

ESADs

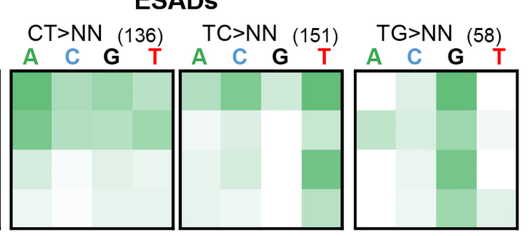

Figure 4. Cisplatin mutational signature in human hepatocellular carcinomas (HCCs) and esophageal adenocarcinomas (ESADs). ( $A$ ) Example SNS and (B) DNS mutational spectra of a tumor that tested positive for the cisplatin signature in the SNS analysis (HK034). In $A$ and $B$, numbers of mutations in each mutation class are indicated. (C) DNS cosine similarities between the experimental cisplatin signature and HCCs, grouped on whether they were negative (left) or positive (right) for cisplatin mutagenesis in the SNS analysis. Red dots represent HCCs that were found positive for cisplatin mutagenesis in the SNS analysis but did not show the cisplatin DNS signature (false-positives) and samples that were not found cisplatin-positive in the SNS analysis but were concluded to be cisplatin-positive based on the DNS analysis (false-negatives). (D) \pm 1 -bp sequence context preferences for the most prominent DNS mutation classes in cisplatin-positive HCCs and ESADs. Total numbers of DNSs per mutation class are indicated in parentheses. The vertical axis is the preceding (5') base, the horizontal axis is the following ( $\left.3^{\prime}\right)$ base. (E) DNS transcription strand bias in all cisplatin-positive tumors combined. For the individual sample plots, see Supplemental Figure S27. (F) DNS replication timing bias in cisplatin-positive HCCs. DNSs were classified as being in either early or late replicating regions as described in Methods.

delineated SNS and DNS signatures in hand, we were able to detect cisplatin mutagenesis in HCCs and ESADs with high confidence. All HCCs for which clinical data were available and all esophageal cancers indeed had histories of prior cisplatin treatment. We therefore conclude that the mutational signature established here serves as a biomarker for cisplatin mutagenesis that could be used to determine whether or not a suspected secondary malignancy was indeed induced by cisplatin.

Prior to this study, two different experimentally elucidated mutational signatures of cisplatin were reported, one in Caenorhabditis elegans and the other in cultured chicken B-cells (DT40) (Meier et al. 2014; Szikriszt et al. 2016). Both studies found primarily 
C > A mutations, but in terms of SNSs in trinucleotide context, the signatures bore no resemblance to each other or to the MCF-10A/HepG2 signature reported here (Supplemental Fig. S28A). In the C. elegans data, this was true for both the DNA repair-proficient worms as well as for all worms combined. Like our experimental data, the exposed worms and DT40 cells had relatively high numbers of DNSs relative to SNSs, and the DT40 DNS spectra closely resembled our experimental DNS signature (cosine similarity $=0.935) \quad($ Supplemental Fig. S28B). However, in neither system was it possible to discern the MCF-10A/HepG2 SNS signature in the mutation spectra, due to the high number of $\mathrm{C}>\mathrm{A}$ mutations (Supplemental Fig. S28A). We also note that the $\mathrm{C}>\mathrm{A}$ mutations in the treated worms and DT40 cells do not resemble any currently known mutational signature or artifact (Forbes et al. 2017; cancer.sanger.ac.uk). In light of the similarity between the MCF-10A/HepG2 and DT40 DNS signatures, we further investigated whether the DT40 SNS signature might be present in HCCs or ESADs. Comparisons using the mSigAct signature presence test concurred that, compared to the DT40 SNS signature, the MCF-10A/HepG2 signature is more effective at detecting cisplatin-mutagenized HCCs and ESADs and at explaining their mutational spectra (Supplemental Data S1).

The differences between the MCF-10A/HepG2 SNS cisplatin signature and the C. elegans and DT40 signatures might stem from the different model organisms used, which may differ in DNA damage susceptibility and characteristics of DNA repair and replication errors. In any case, the differences between the previously published cisplatin spectra and the MCF-10A/HepG2 signature emphasize the need for standardization of in vitro mutational signature models. We propose that it is prudent to use human cell lines for experimental elucidation of mutational signature etiology, to avoid possible differences in trans-lesion synthesis and DNA repair proficiencies between organisms.

Mutational processes reflect the cumulative effect of three steps: (1) DNA damage (for cisplatin, adduct formation); (2) DNA repair (for cisplatin, NER), which may or may not correct the damage; and (3) if DNA repair fails, trans-lesion synthesis across the damaged base or bases, which may replicate the DNA correctly or incorrectly, in the latter instance creating a mutation. In this study, while known patterns of adduct formation did not predict the patterns of substitutions (Fig. 5), we can nevertheless postulate models that explain the observed mutations by combining our knowledge of adduct formation and models of how DNA replication and trans-lesion synthesis might behave (2 and 3).

First, despite high proportions of DNSs relative to SNSs, SNSs still greatly outnumbered the DNSs (Fig. 5A). We postulate that these SNSs are formed by correct trans-lesion synthesis opposite one of the purines of the purine-purine intra-strand crosslinks, and misincorporation occurring opposite the other, as has been shown for UV-induced intra-strand crosslinks (McCulloch et al. 2004). This is supported by the high number of SNSs at potential intra-strand crosslink sites: $85 \%$ of the SNSs are at GpG, GpA, or ApG sites (Supplemental Fig. S29). Closer inspection of SNSs in trinucleotides encompassing only a single potential intra-strand crosslink site revealed that at most such sites, SNSs are more common at the $3^{\prime}$ adducted-base across every cell-line clone (Supplemental Fig. S30). However, at potential adenine-guanine intra-strand crosslink sites, SNSs are more common at the $5^{\prime}$ base. We do not have an explanation for this difference. Possibly different trans-lesion synthesis polymerases are involved in traversing the various intra-strand crosslinks.

Second, the relative abundance of the different types of DNSs did not correspond to the reported ratios of intra-strand and interstrand adducts at their respective dinucleotides (cf. the right piecharts of Fig. 5A,B with graphical representations of the most prominent adducts in Fig. 5C). Of the DNSs, $24.7 \%$ were in potential inter-strand crosslink sites, while these represent $<5 \%$ of cisplatin-adducts (Jamieson and Lippard 1999; Enoiu et al. 2012). However, the higher proportion of DNSs putatively due to interstrand crosslinks is consistent with inter-strand crosslinks being more damaging and harder to repair than intra-strand crosslinks (Andreassen and Ren 2009; Hashimoto et al. 2016; Roy and Scharer 2016).

In this study, combined SNS and DNS information was crucial for high-confidence detection of cisplatin mutagenesis in human tumors. SNS analysis alone would have identified three false-positives and missed RK072 and RK140, and DNSs analysis alone

\section{Genome Research}

www.genome.org 
would have identified several likely false-positives. Ideally, the field of mutational signature analysis will move toward a standard of integrated SNS and DNS analysis. To enable this, a comprehensive catalog of DNS signatures similar to that of SNS signatures (Forbes et al. 2017; cancer.sanger.ac.uk) would be required.

\section{Methods}

\section{Cell line exposure and whole-genome sequencing}

MCF-10A and HepG2 cells were obtained from the ATCC. MCF$10 \mathrm{~A}$ was cultured in DMEM/F12 medium supplemented with $10 \%$ FBS, $10 \mathrm{ng} / \mathrm{mL}$ insulin, $20 \mathrm{ng} / \mathrm{mL}$ EGF, $0.5 \mu \mathrm{g} / \mathrm{mL}$ hydrocortisone, $50 \mathrm{ng} / \mu \mathrm{L}$ penicillin, and $50 \mathrm{U} / \mathrm{mL}$ streptomycin. HepG2 was cultured in minimal essential medium (MEM) supplemented with $10 \% \mathrm{FBS}$, nonessential amino acids, $50 \mathrm{ng} / \mu \mathrm{L}$ penicillin, and $50 \mathrm{U} /$ $\mathrm{mL}$ streptomycin. For cisplatin exposure, 60,000 (MCF-10A) or 250,000 (HepG2) cells/well were seeded at day 0 in a six-well plate. On day 1 , cisplatin was added to final concentrations of 0.5 and 1 $\mu \mathrm{M}$ (MCF-10A) or $0.75 \mu \mathrm{M}$ (HepG2). At day 7, cells were trypsinized, counted, and reseeded in a new six-well plate. This process was repeated eight times. As mutagenesis requires DNA replication, the proliferation rate was monitored (Supplemental Fig. S31). After 4 and $8 \mathrm{wk}$, cells were expanded, and single cells were FACS-isolated directly into a 96-well plate with culture medium. These single-cell clones were expanded for DNA isolation and whole-genome sequencing. In addition, the MCF-10A and HepG2 cell lines were sampled at the start of the cisplatin exposure. DNA isolation was performed using the Wizard Genomic DNA Purification kit (Promega) according to the manufacturer's instructions. Paired-end sequencing was performed on a HiSeq X Ten instrument with 150-bp reads at Novogene Co., Ltd.

\section{Alignment and variant calling}

Read alignment to hs37d5 was done using BWA-MEM, followed by PCR duplicate removal and merging using Sambamba (v0.5.8) (Tarasov et al. 2015). Variant calling was performed using Strelka (v1.014) (Saunders et al. 2012). Variants in dbSNPv132, 1000 genomes (The 1000 Genomes Project Consortium 2015), segmental duplications, microsatellites and homopolymers, and the GL and decoy sequences were excluded. Additionally, variants were filtered for having at least $20 \%$ variant allele frequency, $25 \times$ coverage in both treated and control sample, and at least four reads supporting the variant; $0.4 \%$ and $0.2 \%$ of the variants were shared between the clones from the 0.5 and $1 \mu \mathrm{M}$ treated cells. Supplemental Figure S32 shows the variant allele frequency distribution.

DNSs were identified as two adjacent SNSs. As primary QC we checked that the variant allele frequencies of both SNSs were equal. Second, we recalled the genomes using FreeBayes, which calls DNSs when the SNSs are in the same reads (Garrison and Marth 2012). Out of the 2868 DNSs extracted from the Strelka calls, 2818 were also called by FreeBayes. Last, we checked the DNSs in IGV. All DNSs identified from the Strelka analysis were in the same DNA molecule. Focusing specifically on those DNSs that were not called by FreeBayes, 17 were not called as DNSs by FreeBayes, as they were close to a germline variant, and FreeBayes called these as tri- or tetranucleotide substitutions. Beyond this, 24 putative DNSs were part of complex mutations that were not, in fact, DNSs. Of the remaining nine putative DNSs not called by FreeBayes, five were likely false-positives, as most were only present in one sequence read direction, in regions with low mapping quality, or located near the end of sequencing reads. Overall, we estimated the initial false-discovery rate of DNSs to be $\sim 1.2 \%(33 / 2868)$, but after FreeBayes and IGV inspec- tion we estimate that the false-discovery rate is close to zero. For indels, the intersection between the Strelka and FreeBayes calls was used.

\section{Copy number analysis}

FreeBayes calls were filtered to select variants in dbSNPv132. Coverage and B-allele-frequencies were extracted and segmented using the Quantsmooth (v1.44.0) package in R (Eilers and de Menezes 2005).

\section{Detection of structural variants}

Manta v0.29.6 was used to detect structural variants (SVs) present in the cisplatin-treated but not the untreated samples (Chen et al. 2016). The following filters were applied: (1) Breakpoints of intrachromosomal SVs must be $\geq 1000$ bp apart. (2) Both breakpoints must be located on autosomes. (3) Each candidate SV must be supported by at least 10 spanning or split reads.

\section{Statistical analysis of enrichment of extended sequence context}

To test for enrichment or depletion of SNSs in extended sequence context, we used a binomial test. The null hypothesis was that the proportion of occurrences of a given penta- or heptanucleotide centered on a given SNS (one of $\mathrm{C}>\mathrm{A}, \mathrm{C}>\mathrm{G}, \mathrm{C}>\mathrm{T}, \mathrm{T}>\mathrm{A}, \mathrm{T}>\mathrm{C}$, or $\mathrm{T}>\mathrm{G}$ ) was the same as the proportion of all penta-/heptanucleotides centered on that SNS.

We take as an example a C > T SNS at the center of pentanucleotide TCCAT in the combined MCF-10A data. There were a total of $9509 \mathrm{C}>\mathrm{T}$ mutations in the sequenced portions of the genome, of which 162 were in TCCAT. In total there were $1,089,134,720$ pentanucleotide sites centered on $\mathrm{C}$ in the sequenced regions of the genome, of which $7,046,748$ were TCCAT. We then used the $\mathrm{R}$ function call binom.test $(\mathrm{x}=162, \mathrm{n}=9509, \mathrm{p}=[7,046,748 /$ $1,089,134,720])$, which rejected the null hypothesis with $P<$ $1.13 \times 10^{-26}$.

\section{Analysis of association between cisplatin mutations and genomic features}

We obtained processed ChIP-seq data sets for HepG2 for H3K4me1, H3K4me2, H3K4me3, H3K9ac, H3K9me3, H3K27ac, H3K27me3, H3K36me3, H3K79me3, CTCF, and EZH2 from www.ncbi.nlm. nih.gov/geo/, accession GSE29611. As histone ChIP-seq data for MCF-10A was not available, we obtained analogous data for normal human mammary epithelial cells and used this as a substitute. We obtained MCF-10A expression data from www.ncbi.nlm.nih. gov/geo/, accession GSM1100206, and HepG2 expression data from the NIH Roadmap Epigenomics Mapping Consortium (Bernstein et al. 2010; http://egg2.wustl.edu/roadmap/data/byDataType/rna/ expression/57epigenomes.RPKM.pc.gz). For analysis of replication timing and replication strand bias, we obtained processed replication timing (Repli-Seq) data for HepG2 and MCF7 (MCF-10A data was not available) from GEO (www.ncbi.nlm.nih.gov/geo/, accessions GSM923446 and GSM923442). We determined replication strand according to Liu et al. (2016).

\section{Sources of publicly available sequencing data}

This study used whole-genome sequencing data from 264 HCCs from Japan (Fujimoto et al. 2016) and 78 from Hong Kong (Kan et al. 2013) and 140 ESADs (Noorani et al. 2017). Additionally, we used whole-genome sequencing data of 24 lung adenocarcinomas (Imielinski et al. 2012) and 112 melanomas. For the HCCs, ESADs, and melanomas, simple somatic mutation data was downloaded from the International Cancer Genome Consortium 
(ICGC) data portal (Zhang et al. 2011; https://dcc.icgc.org/, release 18 , March, 2015). The 78 Hong Kong HCCs were re-analyzed as described previously (Huang et al. 2017).

\section{Analysis of the SNS cisplatin signature exposure in tumors}

We used the mSigAct signature presence test (Ng et al. 2017) to assess presence of the experimental cisplatin SNS signature in the publicly available mutational spectra of HCCs and ESADs as specified in Supplemental Data S2. Briefly, the mSigAct signature presence test determines the likelihood of the observed mutation spectrum with and without a contribution from the target signature and compares these with a likelihood ratio test. The null hypothesis is that the counts are generated without a contribution from the target signature, and the alternative hypothesis is that they were generated with a contribution from the target signature. We took the weighted average of the SNS spectra of all MCF-10A and HepG2 cisplatin clones as the cisplatin SNS signature. As described in $\mathrm{Ng}$ et al. (2017), the mSigAct signature presence test has better receiver operating characteristics than the NMF approach from Alexandrov et al. (2013a,b) (LA-NMF for short), as assessed by tests on simulated data. More concretely, the mSigAct signature presence test is better suited for conservative assessment of the presence of a signature. In what follows, we will use the customary notation for NMF, $V \approx W H$, in which $V$ is the matrix of observed mutational spectra, $W$ is the matrix of mutational signatures, and $H$ is the matrix of "exposures." LA-NMF imposes no sparsity constraints on the number of signatures operating in a tumor (i.e., on the number of nonzero elements in columns of $H$ ). As shown in Section 4.3 of Alexandrov (2014), the $W$ and $H$ matrices determined by LA-NMF are sometimes highly variable depending on the specific subset of tumors in $V$, especially when $V$ contains relatively small number of tumors, as is the case for the current study. The mSigAct presence test avoids this problem by using the precomputed consensus signatures computed by LA-NMF to address the narrower question of whether a given signatures is plausibly necessary to account for a single tumor's mutational spectrum. The mSigAct software is available from the URL https:// zenodo.org/record/843773\#.WZQQE1EjHRZ as the following doi: 0.5281/zenodo.843773 ( $\mathrm{Ng}$ et al. 2017). Supplemental Data S3 and S4 detail an alternative analysis that reconstructs spectra using mathematically optimized linear combinations of COSMIC signatures. This alternative analysis then identifies possible cisplatin-exposed tumors based on a cutoff of $>5 \%$ of mutations being attributed to cisplatin. This approach has similar sensitivity to the mSigAct signature presence test (it misses one ESAD), but it is not as specific.

\section{NMF on DNS spectra}

To assess the effect of cisplatin on primary tumors based on DNSs, we developed a customized semi-supervised NMF method that incorporated the method from Schmidt et al. (2007) into the LANMF code from Alexandrov et al. (2013b); Supplemental Data S5 provides the patch file. Again using the notation $V \approx W H$ to describe NMF, ssNMF treats $W$, the signature matrix, as composed of two segments: $W_{f}$, which specifies the known, fixed signatures, and $W_{u}$, which is computed by NMF. ssNMF updates only $W_{u}$ and $H$. The advantage of using ssNMF rather than the closely related method in Alexandrov et al. (2013b) is that ssNMF can directly ask the question: "To what extent can the DNS spectra of sets of HCCs and ESADs be explained by the action of the experimental cisplatin DNS signature combined with a reasonably small number of additional, unknown signatures?" In contrast, NMF would have to rediscover the cisplatin signature, which, because of the inher- ent limitations of LA-NMF, may vary from the experimental signature (Alexandrov 2014). Supplemental Data S6 describes in more detail the advantages of using ssNMF.

We ran ssNMF separately on (1) $V_{\mathrm{HCC}}$, which contained the DNS spectra of the HCCs, lung adenocarcinomas, and the cell line clones, and (2) $V_{\mathrm{ESAD}}$, which contained the DNS spectra of the ESADs and the cell line clones. We ran ssNMF on each of $V_{\mathrm{HCC}}$ and $V_{\mathrm{ESAD}}$, asking for $2,3,4$, and 5 signatures in total. In all cases $W_{f}$ consisted of a fixed signature that was the weighted average of the DNS signatures of MCF-10A and HepG2. Using the signature stability and average Frobenius reconstruction error approach described in Alexandrov et al. (2013b), we chose three signatures for both $V_{\mathrm{HCC}}$ and $V_{\mathrm{ESAD}}$ (Supplemental Figs. S25, S26). As a further sanity check on the ssNMF analyses, we ran analogous analyses using LA-NMF. This yielded very similar results (Supplemental Data S6).

\section{Data access}

The raw sequencing data from this study have been submitted to the European Nucleotide Archive (ENA, http://www.ebi.ac.uk/ ena) under accession number PRJEB21971. The sample accessions are listed in Supplemental Table S1.

\section{Acknowledgments}

We thank Willie Yu for technical assistance. This study was funded by NMRC/CIRG/1422/2015 to S.G.R.

Author contributions: A.B. and S.G.R. designed the study. A.B. and S.-C.H. performed in vitro experiments. A.B., M.N.H., and J.Q.L. carried out electronic analyses. A.W.T.N. provided analysis tools and technical support. Y.K., K.C., and H.N. provided clinical information. A.B. and S.G.R. drafted the manuscript and prepared figures. A.B., B.T.T., and S.G.R. edited the manuscript. All authors read and approved the final manuscript.

\section{References}

The 1000 Genomes Project Consortium. 2015. A global reference for human genetic variation. Nature 526: 68-74.

Alexandrov LB. 2014. "Signatures of mutational processes in human cancer." PhD thesis, Darwin College, University of Cambridge, Cambridge, UK. $\mathrm{ftp}: / / \mathrm{ftp}$. sanger.ac.uk/pub/resources/theses/la2/alexandrov_ludmil_thesis. pdf.

Alexandrov LB, Nik-Zainal S, Wedge DC, Aparicio SA, Behjati S, Biankin AV, Bignell GR, Bolli N, Borg A, Borresen-Dale AL, et al. 2013a. Signatures of mutational processes in human cancer. Nature 500: 415-421.

Alexandrov LB, Nik-Zainal S, Wedge DC, Campbell PJ, Stratton MR. 2013b. Deciphering signatures of mutational processes operative in human cancer. Cell Rep 3: 246-259.

Alexandrov LB, Jones PH, Wedge DC, Sale JE, Campbell PJ, Nik-Zainal S, Stratton MR. 2015. Clock-like mutational processes in human somatic cells. Nat Genet 47: 1402-1407.

Andreassen PR, Ren K. 2009. Fanconi anemia proteins, DNA interstrand crosslink repair pathways, and cancer therapy. Curr Cancer Drug Targets 9: 101-117.

Baik MH, Friesner RA, Lippard SJ. 2003. Theoretical study of cisplatin binding to purine bases: Why does cisplatin prefer guanine over adenine? J Am Chem Soc 125: 14082-14092.

Behmand B, Marignier JL, Mostafavi M, Wagner JR, Hunting DJ, Sanche L. 2015. Radiosensitization of DNA by cisplatin adducts results from an increase in the rate constant for the reaction with hydrated electrons and formation of Pt(I). J Phys Chem B 119: 9496-9500.

Bernstein BE, Stamatoyannopoulos JA, Costello JF, Ren B, Milosavljevic A, Meissner A, Kellis M, Marra MA, Beaudet AL, Ecker JR, et al. 2010. The NIH Roadmap Epigenomics Mapping Consortium. Nat Biotechnol 28: 1045-1048.

Chen X, Schulz-Trieglaff O, Shaw R, Barnes B, Schlesinger F, Kallberg M, Cox AJ, Kruglyak S, Saunders CT. 2016. Manta: rapid detection of structural variants and indels for germline and cancer sequencing applications. Bioinformatics 32: 1220-1222.

\section{Genome Research}

www.genome.org 
Choi DK, Helenowski I, Hijiya N. 2014. Secondary malignancies in pediatric cancer survivors: perspectives and review of the literature. Int J Cancer 135: $1764-1773$.

Conaway JW, Conaway RC. 1999. Transcription elongation and human disease. Annu Rev Biochem 68: 301-319.

Dasari S, Tchounwou PB. 2014. Cisplatin in cancer therapy: molecular mechanisms of action. Eur J Pharmacol 740: $364-378$.

Dugbartey GJ, Peppone LJ, de Graaf IA. 2016. An integrative view of cisplatin-induced renal and cardiac toxicities: molecular mechanisms, current treatment challenges and potential protective measures. Toxicology 371: 58-66.

Eastman A. 1983. Characterization of the adducts produced in DNA by cisdiamminedichloroplatinum(II) and cis-dichloro(ethylenediamine)platinum(II). Biochemistry 22: 3927-3933.

Eilers PH, de Menezes RX. 2005. Quantile smoothing of array CGH data. Bioinformatics 21: 1146-1153.

Enoiu M, Jiricny J, Scharer OD. 2012. Repair of cisplatin-induced DNA interstrand crosslinks by a replication-independent pathway involving transcription-coupled repair and translesion synthesis. Nucleic Acids Res 40: 8953-8964.

Fichtinger-Schepman AM, Vendrik CP, van Dijk-Knijnenburg WC, de Jong WH, van der Minnen AC, Claessen AM, van der Velde-Visser SD, de Groot G, Wubs KL, Steerenberg PA, et al. 1989. Platinum concentrations and DNA adduct levels in tumors and organs of cisplatin-treated LOU/M rats inoculated with cisplatin-sensitive or -resistant immunoglobulin $\mathrm{M}$ immunocytoma. Cancer Res 49: 2862-2867.

Forbes SA, Beare D, Boutselakis H, Bamford S, Bindal N, Tate J, Cole CG, Ward S, Dawson E, Ponting L, et al. 2017. COSMIC: somatic cancer genetics at high-resolution. Nucleic Acids Res 45: D777-D783.

Fousteri M, Mullenders LH. 2008. Transcription-coupled nucleotide excision repair in mammalian cells: molecular mechanisms and biological effects. Cell Res 18: 73-84.

Fujimoto A, Furuta M, Totoki Y, Tsunoda T, Kato M, Shiraishi Y, Tanaka H, Taniguchi H, Kawakami Y, Ueno M, et al. 2016. Whole-genome mutational landscape and characterization of noncoding and structural mutations in liver cancer. Nat Genet 48: 500-509.

Garrison E, Marth G. 2012. Haplotype-based variant detection from shortread sequencing. arXiv:1207.3907 [q-bio.GN.

Harrington CF, Le Pla RC, Jones GD, Thomas AL, Farmer PB. 2010. Determination of cisplatin 1,2-intrastrand guanine-guanine DNA adducts in human leukocytes by high-performance liquid chromatography coupled to inductively coupled plasma mass spectrometry. Chem Res Toxicol 23: 1313-1321.

Hashimoto S, Anai H, Hanada K. 2016. Mechanisms of interstrand DNA crosslink repair and human disorders. Genes Environ 38: 9.

Hu J, Adar S, Selby CP, Lieb JD, Sancar A. 2015. Genome-wide analysis of human global and transcription-coupled excision repair of UV damage at single-nucleotide resolution. Genes Dev 29: 948-960.

Hu J, Lieb JD, Sancar A, Adar S. 2016. Cisplatin DNA damage and repair maps of the human genome at single-nucleotide resolution. Proc Natl Acad Sci 113: 11507-11512.

Huang MN, Yu W, Teoh WW, Ardin M, Jusakul A, Ng AWT, Boot A, AbediArdekani B, Villar S, Myint SS, et al. 2017. Genome-scale mutational signatures of aflatoxin in cells, mice, and human tumors. Genome Res 27: 1475-1486.

Imielinski M, Berger AH, Hammerman PS, Hernandez B, Pugh TJ, Hodis E, Cho J, Suh J, Capelletti M, Sivachenko A, et al. 2012. Mapping the hallmarks of lung adenocarcinoma with massively parallel sequencing. Cell 150: $1107-1120$.

Jamieson ER, Lippard SJ. 1999. Structure, recognition, and processing of cisplatin-DNA adducts. Chem Rev 99: 2467-2498.

Kaiser VB, Taylor MS, Semple CA. 2016. Mutational biases drive elevated rates of substitution at regulatory sites across cancer types. PLoS Genet 12: e1006207.

Kan Z, Zheng H, Liu X, Li S, Barber TD, Gong Z, Gao H, Hao K, Willard MD, $\mathrm{Xu}$ J, et al. 2013. Whole-genome sequencing identifies recurrent mutations in hepatocellular carcinoma. Genome Res 23: 1422-1433.

Kushner BH, Cheung NK, Kramer K, Heller G, Jhanwar SC. 1998. Neuroblastoma and treatment-related myelodysplasia/leukemia: the Memorial Sloan-Kettering experience and a literature review. J Clin Oncol 16: 3880-3889.

Liu F, Ren C, Li H, Zhou P, Bo X, Shu W. 2016. De novo identification of replication-timing domains in the human genome by deep learning. Bioinformatics 32: 641-649.

Masters JR, Koberle B. 2003. Curing metastatic cancer: lessons from testicular germ-cell tumours. Nat Rev Cancer 3: 517-525.

McCulloch SD, Kokoska RJ, Masutani C, Iwai S, Hanaoka F, Kunkel TA. 2004. Preferential cis-syn thymine dimer bypass by DNA polymerase eta occurs with biased fidelity. Nature 428: 97-100.
Meier B, Cooke SL, Weiss J, Bailly AP, Alexandrov LB, Marshall J, Raine K, Maddison M, Anderson E, Stratton MR, et al. 2014. C. elegans whole-genome sequencing reveals mutational signatures related to carcinogens and DNA repair deficiency. Genome Res 24: 1624-1636.

Ng AWT, Poon SL, Huang MN, Lim JQ, Boot A, Yu W, Suzuki Y, Thangaraju S, Ng CCY, Tan P, et al. 2017. Aristolochic acids and their derivatives are widely implicated in liver cancers in Taiwan and throughout Asia. Sci Transl Med 9: eaan6446.

Noorani A, Bornschein J, Lynch AG, Secrier M, Achilleos A, Eldridge M, Bower L, Weaver JMJ, Crawte J, Ong CA, et al. 2017. A comparative analysis of whole genome sequencing of esophageal adenocarcinoma preand post-chemotherapy. Genome Res 27: 902-912.

Olivier M, Weninger A, Ardin M, Huskova H, Castells X, Vallee MP, McKay J, Nedelko T, Muehlbauer KR, Marusawa H, et al. 2014. Modelling mutational landscapes of human cancers in vitro. Sci Rep 4: 4482.

Polak P, Karlic R, Koren A, Thurman R, Sandstrom R, Lawrence MS, Reynolds A, Rynes E, Vlahovicek K, Stamatoyannopoulos JA, et al. 2015. Cell-oforigin chromatin organization shapes the mutational landscape of cancer. Nature 518: 360-364.

Poon SL, Pang ST, McPherson JR, Yu W, Huang KK, Guan P, Weng WH, Siew EY, Liu Y, Heng HL, et al. 2013. Genome-wide mutational signatures of aristolochic acid and its application as a screening tool. Sci Transl Med 5: 197ra101.

Ratain MJ, Kaminer LS, Bitran JD, Larson RA, Le Beau MM, Skosey C, Purl S, Hoffman PC, Wade J, Vardiman JW, et al. 1987. Acute nonlymphocytic leukemia following etoposide and cisplatin combination chemotherapy for advanced non-small-cell carcinoma of the lung. Blood 70: 14121417.

Reardon JT, Vaisman A, Chaney SG, Sancar A. 1999. Efficient nucleotide excision repair of cisplatin, oxaliplatin, and Bis-aceto-ammine-dichlorocyclohexylamine-platinum(IV) (JM216) platinum intrastrand DNA diadducts. Cancer Res 59: 3968-3971.

Roy U, Scharer OD. 2016. Involvement of translesion synthesis DNA polymerases in DNA interstrand crosslink repair. DNA Repair (Amst) 44: 33-41.

Saunders CT, Wong WS, Swamy S, Becq J, Murray LJ, Cheetham RK. 2012. Strelka: accurate somatic small-variant calling from sequenced tumornormal sample pairs. Bioinformatics 28: 1811-1817.

Schmidt MN, Larsen J, Hsiao FT. 2007. Wind noise reduction using nonnegative sparse coding. In 2007 IEEE workshop on machine learning for signal processing, pp. 431-436, Thessaloniki, Greece. doi: 10.1109/ MLSP.2007.4414345.

Seplyarskiy VB, Bazykin GA, Soldatov RA. 2015. Polymerase $\zeta$ activity is linked to replication timing in humans: evidence from mutational signatures. Mol Biol Evol 32: 3158-3172.

Szikriszt B, Poti A, Pipek O, Krzystanek M, Kanu N, Molnar J, Ribli D, Szeltner Z, Tusnady GE, Csabai I, et al. 2016. A comprehensive survey of the mutagenic impact of common cancer cytotoxics. Genome Biol 17: 99.

Tarasov A, Vilella AJ, Cuppen E, Nijman IJ, Prins P. 2015. Sambamba: fast processing of NGS alignment formats. Bioinformatics 31: 2032-2034.

Travis LB, Fossa SD, Schonfeld SJ, McMaster ML, Lynch CF, Storm H, Hall P, Holowaty E, Andersen A, Pukkala E, et al. 2005. Second cancers among 40,576 testicular cancer patients: focus on long-term survivors. J Natl Cancer Inst 97: 1354-1365.

Waseem M, Bhardwaj M, Tabassum H, Raisuddin S, Parvez S. 2015. Cisplatin hepatotoxicity mediated by mitochondrial stress. Drug Chem Toxicol 38: 452-459.

Zamble DB, Mu D, Reardon JT, Sancar A, Lippard SJ. 1996. Repair of cisplatin-DNA adducts by the mammalian excision nuclease. Biochemistry 35: 10004-10013.

Zen C, Zen Y, Mitry RR, Corbeil D, Karbanova J, O'Grady J, Karani J, Kane P, Heaton N, Portmann BC, et al. 2011. Mixed phenotype hepatocellular carcinoma after transarterial chemoembolization and liver transplantation. Liver Transpl 17: 943-954.

Zhang J, Baran J, Cros A, Guberman JM, Haider S, Hsu J, Liang Y, Rivkin E, Wang J, Whitty B, et al. 2011. International Cancer Genome Consortium Data Portal-a one-stop shop for cancer genomics data. Database (Oxford) 2011: bar026.

Zhivagui M, Ardin M, Ng AWT, Churchwell M, Pandey M, Villar S, Cahais V, Robitaille A, Bouaoun L, Heguy A, et al. 2018. Experimental analysis of exome-scale mutational signature of glycidamide, the reactive metabolite of acrylamide. bioRxiv doi: 10.1101/254664.

Received September 17, 2017; accepted in revised form March 13, 2018. 


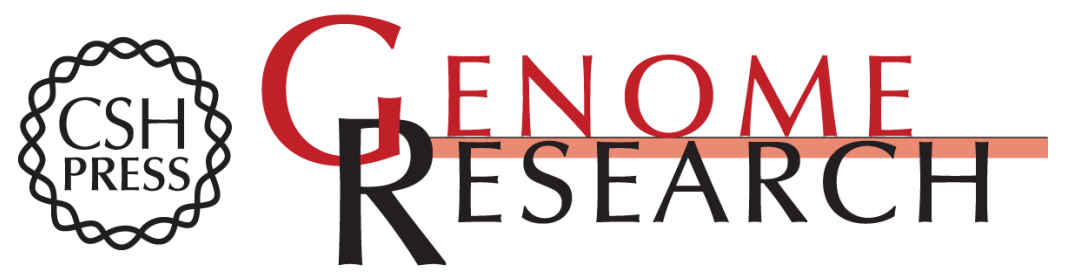

\section{In-depth characterization of the cisplatin mutational signature in human cell lines and in esophageal and liver tumors}

Arnoud Boot, Mi Ni Huang, Alvin W.T. Ng, et al.

Genome Res. 2018 28: 654-665 originally published online April 9, 2018

Access the most recent version at doi:10.1101/gr.230219.117

Supplemental Material

References

Creative

Commons

License

Email Alerting

Service
http://genome.cshlp.org/content/suppl/2018/04/09/gr.230219.117.DC1

This article cites 52 articles, 13 of which can be accessed free at: http://genome.cshlp.org/content/28/5/654.full.html\#ref-list-1

This article is distributed exclusively by Cold Spring Harbor Laboratory Press for the first six months after the full-issue publication date (see

$\mathrm{http}: / /$ genome.cshlp.org/site/misc/terms.xhtml). After six months, it is available under a Creative Commons License (Attribution-NonCommercial 4.0 International), as described at http://creativecommons.org/licenses/by-nc/4.0/.

Receive free email alerts when new articles cite this article - sign up in the box at the top right corner of the article or click here.

\section{Affordable, Accurate Sequencing.}

To subscribe to Genome Research go to:

https://genome.cshlp.org/subscriptions 